\title{
The ins and outs of cyclic di-GMP signaling in Vibrio cholerae
}

\author{
Jenna G. Conner ${ }^{\mathrm{a}}$, David Zamorano-Sánchez ${ }^{\mathrm{a}}$, Jin Hwan Park ${ }^{\mathrm{a}}$, Holger Sondermann ${ }^{\mathrm{b}}$, \\ and Fitnat $\mathrm{H}$. Yildiz ${ }^{*}$ \\ Department of Microbiology and Environmental Toxicology, University of Santa Cruz, \\ Santa Cruz, California, USA ${ }^{a}$ \\ Department of Molecular Medicine, College of Veterinary Medicine, Cornell University, \\ Ithaca, New York, USA ${ }^{\mathrm{b}}$ \\ ${ }^{*}$ Corresponding author: fyildiz@ucsc.edu \\ Short title: c-di-GMP signaling in Vibrio cholerae \\ Key words: Vibrio cholerae; c-di-GMP; biofilm; motility; virulence
}




\begin{abstract}
1 Abstract
2

3 The second messenger nucleotide cyclic dimeric guanosine monophosphate (c-di4 GMP) governs many cellular processes in the facultative human pathogen Vibrio 5 cholerae. This organism copes with changing environmental conditions in aquatic 6 environments and during transitions to and from human hosts. Modulation of c-di-GMP 7 allows $V$. cholerae to shift between motile and sessile stages of life, thus allowing 8 adaptation to stressors and environmental conditions during its transmission cycle. The $9 V$. cholerae genome encodes a large set of proteins predicted to degrade and produce 10 c-di-GMP. A subset of these enzymes has been demonstrated to control cellular 11 processes - particularly motility, biofilm formation, and virulence - through 12 transcriptional, post-transcriptional, and translational mechanisms. Recent studies have 13 identified and characterized enzymes that modulate or sense c-di-GMP levels and have 14 lead towards mechanistic understanding of c-di-GMP regulatory circuits in $V$. cholerae.
\end{abstract}




\section{Introduction}

Bacteria use a range of signal transduction systems to sense environmental conditions and launch adaptive responses, enabling them to enhance survival and proliferation by conserving energy. The signaling nucleotide bis (3'-5') cyclic dimeric guanosine monophosphate (c-di-GMP) has been identified in all major bacterial phyla, and it functions as a second messenger in signal transduction pathways. C-di-GMP is produced by diguanylate cyclases (DGCs), which have GGDEF domains, and degraded by phosphodiesterases (PDEs), which have EAL or HD-GYP domains (Fig 1). The abundance of c-di-GMP metabolizing enzymes, particularly in organisms with complex life cycles, suggests that different c-di-GMP regulatory circuits are activated to regulate cellular processes in response to multiple external cues. The facultative human pathogen Vibrio cholerae, the causative agent of the diarrheal disease cholera, has a complex life cycle involving transitions between aquatic environments and human hosts. The $V$. cholerae genome boasts a high number of predicted DGC and PDE genes, indicating that $\mathrm{c}$-di-GMP may be regulated in response to a variety of fluctuating environmental parameters during its transmission cycle [1]. In this organism, c-di-GMP promotes motile to sessile transition by repressing motility and enhancing biofilm matrix production. Additionally, c-di-GMP represses $V$. cholerae virulence. Below, we outline current knowledge and recent advances regarding the mechanisms by which c-di-GMP participates in regulation of these cellular processes, as well as how external cues are sensed and incorporated into c-di-GMP regulatory circuits.

\section{C-di-GMP is a key repressor of motility}

While the precise molecular mechanisms by which c-di-GMP affects motility in $V$. cholerae are unclear; high c-di-GMP levels inhibit the production and function of $V$. cholerae's single polar flagellum [2,3]. Systematic analysis of the impact of C-di-GMPgenerating and -degrading enzymes on motility using soft agar motility plates (LB $0.3 \%$ agar) at $30^{\circ} \mathrm{C}$ revealed that the absence of the DGCs CdgD (VCA0697), $\mathrm{CdgH}$ (VC1067), CdgK (VC1104), or CdgL (VC2285) enhances motility [4]. Additionally, loss of VpvC (VC2454) leads to increased motility in strains with high c-di-GMP levels [5]. In contrast, lack of predicted PDEs RocS (VC0653) or CdgJ (VC0137) represses motility $[4,6]$. Lack of some of the c-di-GMP metabolizing enzymes impacts motility phenotype without impacting flagellum production, suggesting that c-di-GMP affects the function of the polar flagellum [4].

Cellular c-di-GMP levels vary in different $V$. cholerae isolates [7]. Thus, when tested in different $V$. cholerae genetic backgrounds, additional DGCs and PDEs were shown to govern motility. For example, in the classical biotype, the DGC AcgB (VC1593) and the PDE AcgA (VC1592) regulate motility in low phosphate conditions [8]. The PDE VieA (VC1652) enhances motility in the classical biotype, but not in El Tor [9].

C-di-GMP receptor proteins with PilZ domains play roles in controlling motility. The PilZ domain was the first c-di-GMP binding domain to be characterized, and it has a 
widespread distribution among bacteria, similar to distributions of GGDEF and EAL domains [10]. The PilZ domain has two consensus motifs, RxxxR and D/NxSxxG, required for c-di-GMP binding [11], and it is found in several proteins affecting flagellar motility [12-15]. The $V$. cholerae genome encodes five proteins with predicted PilZ domains: PlzA (VC0697), PlzB (VC1885), PlzC (VC2344), PlzD (VCA0042), and PlzE (VCA0735). All of these Plz proteins except PlzB have the two consensus motifs involved in PilZ c-di-GMP binding. PlzC, PlzD, and PlzE were demonstrated to bind c-diGMP in vitro $[16,17]$. Furthermore, a structural study revealed that PlzD is found as a dimer in the absence of c-di-GMP, and conformational change occurs upon c-di-GMP binding (Fig 2) [18]. The biological consequences of such conformational changes remain unknown. Analyses of strains lacking $\mathrm{Plz}$ proteins have revealed moderate motility phenotypes; PlzB and $\mathrm{PlzC}$ serve as enhancers of motility, while PlzD is a repressor (Fig $3 \mathrm{~A}$ ) $[4,16]$. The molecular basis of motility regulation by Plz proteins in $V$. cholerae is not yet understood.

At the transcriptional level, c-di-GMP represses transcription of flagellar genes. A large set of proteins is required for a functional flagellum, and their expression is regulated by a well-characterized four level transcriptional hierarchy [19]. Transcriptional activation begins with the alternative sigma factor $\sigma^{54}$-dependent regulator FIrA (VC2137). C-di-GMP binds to FlrA and alters its activity, impairing FIrA's ability to activate the expression of the flrBC operon [2]. FlrA is an orthologue of FleQ, the master regulator of flagellar gene expression in Pseudomonas aeruginosa [20]. Structural studies showed that FleQ binds to an intercalated dimer of c-di-GMP and identified three conserved sequence motifs (R-switch $\left(L F R^{144} S\right)$, post-Walker $A\left(R^{185}, N^{186}\right)$ and the $\operatorname{ExxR}^{334}$ ) that contribute to c-di-GMP binding, and all of which are conserved in FIrA [21]. Binding of c-di-GMP to FleQ imposes a change in its oligomeric conformation and acts as an allosteric inhibitor of its ATPase activity. It is yet to be determined if FlrA undergoes similar conformational changes when bound to c-di-GMP. In addition to FIrA, overproduction of the c-di-GMP-dependent transcriptional activator VpsT represses expression of flagellar genes, however the molecular details of this regulation are still being explored [22].

\section{C-di-GMP governs motile-to-sessile life transition and biofilm matrix production}

A biofilm is a surface-attached community of bacteria surrounded in a selfsecreted protective matrix composed primarily of polysaccharides and proteins. Biofilm formation is critical for the survival and infection cycle of $V$. cholerae. This process begins with attachment of cells to surfaces, followed by cell division and production of biofilm matrix components. Major components of the $V$. cholerae biofilm are Vibrio polysaccharide (VPS), which is produced by proteins encoded in the vps-I and vps-II gene clusters, as well as matrix proteins (RbmA, RbmC, and Bap1) that facilitate cellcell and cell-surface interactions and contribute to biofilm architecture [23-25]. C-diGMP regulates both initial attachment and matrix production. 
Systematic analysis of biofilm phenotypes and vps gene expression of $V$. cholerae isogenic mutants with in-frame deletions of predicted DGCs or PDEs found that the DGCs CdgA (VCA0074), CdgH, CdgK, CdgL, CdgM (VC1376), and VpvC and the PDEs RocS, CdpA (VC0130), CdgJ, MbaA (VC0703), CdgC (VCA0785), and VC1851 regulate biofilm formation [5,26]. Additionally, when supplied on a multicopynumber plasmid, VCA0939 induces biofilm formation in a quorum sensing-dependent manner [27]. The PDE VieA represses biofilm formation in the classical biotype, but not El Tor [9].

Biofilm formation is initiated by surface attachment, which is controlled by the MshA pilus. This pilus is assembled via polymerization of MshA subunits, and this assembly requires MshE (VC0405), an AAA+ ATPase that extends the pilus using energy from ATP hydrolysis (Fig 3B). A recent study showed that polymerization of MshA subunits is promoted by C-di-GMP binding via the ATPase MshE $[17,28]$. MshE binds C-di-GMP in its N-terminal domain $[18,29]$. Furthermore, structure of the MshE Nterminal domain revealed that c-di-GMP binds to the unique binding motifs consisting of a tandem array of two highly conserved 24-residue sequence

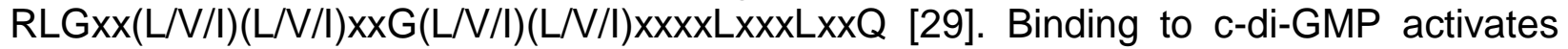
MshE to polymerize the MshA pili, which facilitates attachment. Loss of $m s h E$ or $m s h A$ abolishes initial surface attachment, indicating that c-di-GMP-mediated synthesis of MshA pili is a critical early step in biofilm formation [29].

Biofilm matrix production is regulated by c-di-GMP at the transcriptional level by two key transcriptional activators, VpsR (VC0665) and VpsT (VCA0952) [30] (Fig 3C). VpsR directly activates the expression of VPS biosynthesis genes and genes encoding matrix proteins [31]. The domain architecture of $V p s R$ is similar to members of the Enhancer Binding Protein (EBPs) family. It harbors an atypical REC domain at the $\mathrm{N}$ terminus, a central AAA+ domain, and a Fis-like Helix Turn Helix DNA binding domain at the $\mathrm{C}$-terminus. It has been shown through filter-binding assays that VpsR binds c-diGMP in vitro, with a dissociation coefficient of $1.6 \mu \mathrm{M}$ [32]. Even though c-di-GMP was shown to be dispensable for DNA binding by VpsR in vitro [32], it is possible that coordination of this second messenger plays a role in the interaction of VpsR with the RNA polymerase or induces conformational changes that promote transcription activation. While VpsR shares sequence similarity with FleQ and FIrA, the AAA+ central domain of VpsR lacks the $\sigma^{54}$ binding motif (GAFTGA domain) as well as the conserved residues present in FleQ/FIrA orthologues that form the c-di-GMP binding pocket [21]. This would suggest that the mechanism of c-di-GMP binding, and perhaps the conformational changes imposed by binding of c-di-GMP, are different in VpsR-like proteins.

While $\mathrm{VpsR}$ is required for expression of biofilm genes, VpsT plays an accessory role; loss of vpsT results in decreased expression of vps genes and matrix protein genes [31,33-35]. It has been demonstrated that a dimer of VpsT binds to intercalated c-di-GMP dimer with an affinity of $3.2 \mu \mathrm{M}$ [22]. Structural studies revealed that VpsT has an atypical REC domain with an additional a helix (a6) compared to canonical REC 
domains with a $(\alpha / \beta)_{5}$-fold [22]. The $\alpha 6$ is part of the $c$-di-GMP dependent dimerization interface, where four amino acid residues with the consensus sequence W[F/L/M][T/S]R, form the nucleotide binding pocket. C-di-GMP binding stabilizes the VpsT dimer conformation (Fig 2). Interestingly, VpsT can undergo c-di-GMPindependent dimerization as well. On the basis of genetic and biochemical studies, it has been proposed that this dimerization interface negatively regulates VpsT activity in the absence of c-di-GMP [22].

\section{C-di-GMP regulates the type VI secretion system}

Another $V$. cholerae cellular process that involves c-di-GMP signaling is the type VI secretion system (T6SS). The T6SS is found in many Gram negative bacteria; it is a contractile structure that injects toxic effector proteins upon contact with neighboring prokaryotic or eukaryotic cells [36-38]. C-di-GMP is involved in a T6SS regulatory network via one of $V$. cholerae's two c-di-GMP riboswitches.

Riboswitches are cis-acting elements in the 5' UTR of some mRNA transcripts. In response to a signal, structural rearrangement of riboswitches controls translation or transcriptional termination of a gene product [39,40]. Two types of c-di-GMP riboswitches have been identified in bacteria, class I and class II [41,42]. Class I riboswitches contain a GEMM motif, which is often found in the 5' UTR of bacterial genes with roles in environment, membrane, or motility functions, including many c-diGMP signaling genes [41]. V. cholerae encodes two class I riboswitches, Vc1 and Vc2.

Vc1 is found in the 5' UTR of the gbpA (VCA0811) transcript, which encodes the GlcNAc-binding protein GbpA, a key factor in attachment to surfaces containing Nacetyl-D-glucosamine (GlcNAc) such as intestinal mucus and chitin $[41,43]$. C-di-GMP activates production of GbpA via binding to $\mathrm{Vc}_{\mathrm{c}}$ [44] (Fig 3D).

Vc2 binds c-di-GMP with an affinity of $1 \mathrm{nM}$ and lies upstream of tfoY (VC1722) a master regulator of the T6SS. Studies performed in E. coli, designed to evaluate activity of $\mathrm{V} c 2$ revealed that it can function as an on-switch [41]. Interestingly, a recent report showed increased abundance of TfoY in $V$. cholerae when levels of c-di-GMP are low [45]. The sensitivity of Vc2 to concentrations of c-di-GMP naturally found in $V$. cholerae cells and the mechanism by which $V_{c} 2$ functions remains to be fully unveiled.

\section{C-di-GMP represses virulence}

C-di-GMP represses virulence in $V$. cholerae. The VieSAB three-component system, which regulates C-di-GMP concentration through the phosphodiesterase VieA, is critical for $V$. cholerae virulence. The kinase VieS phosphorylates VieA's REC domain, activating VieA's PDE activity and instigating degradation of c-di-GMP [46]. VieB acts as an inhibitor of VieS and prevents activation of VieA [47]. Loss of the VieSAB system prevents full expression of the gene encoding key virulence regulator ToxT, as well as the genes encoding cholera toxin (CT) [48]. Contact with mammalian epithelial cells promotes expression of vieA, and the EAL domain of VieA was shown to 
be involved in depleting c-di-GMP levels and inducing virulence gene expression upon sensing host cell contact [49].

It is important to note that this regulation is strain-specific. In the classical $V$. cholerae biotype, high c-di-GMP levels lower the amount of ToxT, which in turn lowers virulence gene expression (Fig 3E). El Tor ToxT levels are not altered by high c-di-GMP in vitro; however, there is recent evidence that ToxT does respond to artificially high cdi-GMP in vivo in El Tor strains [32,50]. Hence, the relationship between c-di-GMP and virulence in the El Tor strains remains unclear.

Analysis of gene expression during late stage $V$. cholerae infection revealed that expression of several DGC and PDE genes are induced during late stage infection. These genes encode the DGCs VC2697, VC1593, and VC2370, and the PDE CdpA [51]. While deletion of these genes individually did not significantly impact colonization or transmission phenotypes, a $\Delta V C 2697 \Delta V C 1593 \Delta V C 2370$ triple mutant had a 2-3fold survival defect in stool and pond environments, suggesting that they play a role in transition from host to environment [51] (Fig 3F).

\section{Environmental Signals and Regulation of DGCs and PDEs}

Many $V$. cholerae DGCs/PDEs have sensory domains, suggesting that their activity is controlled by external cues, allowing for c-di-GMP levels to be modulated by environmental parameters. Below, we highlight some of the environmental signals that are known impact cellular c-di-GMP levels (Fig 4).

$V$. cholerae must cope with significant shifts in temperature, both during its residence in aquatic habitats and during transitions between mammalian hosts and the external environment. A recent study showed that low growth temperature enhances cdi-GMP concentration and biofilm formation in $V$. cholerae [52]. Intracellular c-di-GMP concentration was highest in cells grown at $15^{\circ} \mathrm{C}$, followed by $25^{\circ} \mathrm{C}$ and then $37^{\circ} \mathrm{C}$. This temperature-dependent regulation is facilitated by the additive contributions of six diguanylate cyclases: $\mathrm{CdgA}, \mathrm{CdgH}, \mathrm{CdgK}, \mathrm{CdgL}, \mathrm{CdgM}$, and VpvC. Temperature was shown to regulate expression of five of the six DGC genes; however, this transcriptional regulation only partially accounts for the increase in c-di-GMP after shifting growth temperature from $37^{\circ} \mathrm{C}$ to $15^{\circ} \mathrm{C}$, indicating that additional post-transcriptional regulation also contributes to increased c-di-GMP at high temperatures.

In the intestinal lumen, $V$. cholerae is exposed to bile acids which leads to an increase in cellular c-di-GMP concentration [53,54]. The effect of bile acids on c-di-GMP is mediated by three DGCs, CdgH, VC1372, and CdgM, and one PDE, VC1295 [54]. Bile acids regulate the DGCs at the post-transcriptional level and the PDE at the transcription level through unknown mechanisms. Two of the DGCs, CdgH and CdgM, possess sensory domains that may be involved in sensing bile acids. Interestingly, $\mathrm{CdgH}$ and $\mathrm{CdgM}$ are also involved in the response to temperature, indicating that these proteins are part of multiple c-di-GMP signaling pathways and may be capable of sensing multiple signals [52]. The effect of bile acids on c-di-GMP is quenched by bicarbonate, which causes a local increase in $\mathrm{pH}$ [54]. These findings indicate a 
scenario in which $V$. cholerae c-di-GMP concentration is finely-tuned and dependent on the cells' precise location within the host intestine.

Polyamines, which are found within the human gastrointestinal tract, represent another signal modulating cellular c-di-GMP concentration in $V$. cholerae. The sensor protein NspS regulates activity of a PDE in response to polyamines. Two structurallysimilar polyamines, norspermidine and spermidine, regulate $V$. cholerae c-di-GMP and biofilm formation [55]. Exogenous norspermidine activates $V$. cholerae biofilm formation through a pathway involving the membrane-bound PDE MbaA and its periplasmic partner protein NspS, which are encoded in the same operon [56]. Evidence suggests that NspS binds norspermidine and then interacts with the periplasmic domain of MbaA and inhibits its PDE activity, thus preventing repression of biofilm genes. Conversely, exogenous spermidine was shown to repress $V$. cholerae biofilm formation; this repression may occur via competition with norspermidine for the NspS binding site $[57,58]$.

In addition to signals modulating cellular c-di-GMP levels, recent studies revealed mechanisms of activation of DGCs via environmental signals. The $V$. cholerae enzyme Vc Bhr-DGC (VC1216) possesses a Bhr domain, which can bind two non-heme di-iron atoms in either ferrous or ferric form [59]. Binding to ferrous di-iron enhances the DGC activity of the enzyme, supporting a model in which Vc Bhr-DGC stimulates c-di-GMP concentration and biofilm formation in anoxic environments.

\section{Conclusion}

C-di-GMP-mediated phenotypes are critical for the life cycles of many bacteria, including the human pathogen $V$. cholerae. Therefore, understanding the inputs that influence c-di-GMP production and degradation and the consequences of c-di-GMP signaling is crucial. Significant gains in our knowledge of the regulation of c-di-GMP and the role of c-di-GMP-mediated phenotypes in $V$. cholerae have been made. We now understand mechanisms of action of DGCs and PDEs and key transcriptional regulators in c-di-GMP signaling, as well as the impact of many environmental cues on c-di-GMP signaling. Due to versatility of the c-di-GMP structure, c-di-GMP-binding proteins employ a wide variety of binding motifs and mechanisms of action, making them difficult to predict computationally. While novel screening methods have facilitated identification of new receptors, the roles of these proteins in $V$. cholerae biology are yet to be determined [17]. Further studies are needed to reveal the mechanisms of action of known c-di-GMP binding proteins, such as the Plz proteins, as well as to determine if any c-di-GMP effectors remain to be discovered in $V$. cholerae. Additionally, it is not yet known how c-di-GMP regulates virulence factors, or how c-di-GMP's regulation of biofilm and motility contribute to infection. Further characterization of environmental signals, receptors, and specificity in c-di-GMP signaling will greatly inform our understanding of how bacteria adapt to environmental stressors, and may allow for development of novel strategies to cope with problems posed by biofilm, such as biofouling and antibiotic resistance. 


\section{Figure Legends}

Fig 1. Model of c-di-GMP signaling. C-di-GMP is synthesized from GTP by diguanylate cyclases (DGCs) harboring catalytic GGDEF domains and degraded by phosphodiesterases (PDEs), which can have EAL or HD-GYP catalytic domains. Bacteria often possess multiple DGCs and PDEs; furthermore, many DGCs and PDEs are multi-domain proteins harboring sensing domains, indicating that their activity may be regulated by environmental signals. The oligoribonuclease Orn regulates the abundance of $p G p G$. Elevated levels of $p G p G$ are responsible for product inhibition of EAL containing enzymes in Pseudomonas aeruginosa ([60,61]). The $V$. cholerae genome is encodes 31 GGDEF domain, 12 EAL domain, 10 GGDEF-EAL domain, and 9 HD-GYP domain. Cellular c-di-GMP molecules are sensed by multiple types of receptors that regulate downstream cellular processes at transcriptional, posttranscriptional, and post-translational levels.

Fig. 2. Structure of c-di-GMP receptors. (A) PilZ domain-containing protein PlzD. Constitutive dimerization of PlzD is mediated by its $\mathrm{YcgR}-\mathrm{N}^{*}$ domain (green), which adopts the same fold as its PilZ domain (purple). Binding of c-di-GMP to a site involving the linker and the PilZ domain induces an intramolecular conformational change. PDB codes: 1yln [Zhang et al., unpublished]; 2rde [18]. (B) Transcription factor VpsT. The dinucleotide stabilizes a dimer between two VpsT molecules. The dimerization interface is characterized by a signature helix that complements the fold of this subfamily of receiver domains (pink). PDB codes: 3kln and 3klo [22]. (C) MshEN domain-containing proteins. MshE-type ATPases contain the conserved the MshEN domain that binds c-diGMP through an extended sequence motif in its MshEN_N domain (purple). PDB code: 5htl [29]. (D) RNA aptamers. A class of c-di-GMP-binding riboswitches has been identified as a downstream target of the dinucleotide. Helices P1, P2, and P3 are colored in shades of blue; nucleotides that interact with the nucleotide directly are shown in pink. PDB code: 3irw [52].

Fig. 3. C-di-GMP signaling modules. (A) The DGCs CdgD, CdgH, CdgL, and CdgK, and the PDEs RocS and CdgJ, regulate a pool of c-di-GMP that contributes to $\mathrm{V}$. cholerae motility. This $c-d i-G M P$ is then bound by receptors that regulate motility. Evidence suggests that the PilZ proteins PIzB, PlzC, PlzD, and PlzE bind c-di-GMP and may interact with flagellar motor proteins to control motility. The transcription factor FlrA binds c-di-GMP and controls expression of the flagellar hierarchy.

(B) The PDEs RocS and CdgJ, along with an uncharacterized DGC(s), regulate a pool of c-di-GMP that controls $V$. cholerae surface attachment. C-di-GMP is bound by the receptor protein MshE, which forms a hexameric ring and controls extension of the MshA pilus. Upon binding c-di-GMP, MshE triggers assembly of the MshA pilus in a process involving the MSHA biogenesis proteins MshG, MshL, and the major pilin MshA (shown in blue).

(C) The DGCs VpvC, CdgA, CdgH, CdgL, CdgK, and VCA0930, and the PDEs RocS, MbaA, CdgC, CdpA, VC1851, and CdgJ regulate c-di-GMP levels and regulate $V$. 
cholerae biofilm formation. C-di-GMP is bound by the transcription factors VpsT and VpsR. C-di-GMP binding stabilizes VpsT dimers and allows for enhanced expression of biofilm genes. The function of VpsR binding to c-di-GMP remains unclear.

(D) DGCs and/or PDEs regulate levels of c-di-GMP bound by the riboswitches $V_{c} 1$ and Vc2. Upon c-di-GMP binding, Vc1 allows translation of GbpA, a critical virulence factor. GbpA facilitates binding to surfaces with GlcNAc, such as intestinal mucus and chitin. $\mathrm{Vc2}$ regulates translation of TfoY, which then activates the type $\mathrm{VI}$ secretion system (T6SS). It is not yet clear whether c-di-GMP allows or prevents translation of tfoY.

(E) The PDE VieA, along with an uncharacterized $\mathrm{DGC}(\mathrm{s})$, regulates virulence by degrading C-di-GMP, which then reduces production of the virulence regulator ToxT. ToxT regulates expression of the genes required to produce cholera toxin (CT).

Fig. 4. Model for signals modulating c-di-GMP levels. (A) In the aquatic environment, $V$. cholerae experiences shifts in temperature and likely in oxygen availability. High temperatures $\left(30^{\circ} \mathrm{C}\right)$ repress c-di-GMP levels through six DGCs; CdgA, CdgL, $\mathrm{CdgH}$, CdgM, CdgK, and VpvC. Likewise, low temperature $\left(15^{\circ} \mathrm{C}\right)$ enhances C-di-GMP production through the same six DGCs. High oxygen levels, such as those expected in the outer edges of mature biofilms, contribute to formation of ferric iron, which can be bound by Vc Bhr-DGC. Upon binding two molecules of ferric iron, Vc Bhr-DGC's activity is repressed. Low oxygen levels, such as those expected deeper inside of a mature biofilm, contribute to formation of ferrous iron. Vc Bhr-DGC activity is enhanced when bound to two molecules of ferrous iron, supporting a model in which $V$. cholerae biofilm production is enhanced in anoxic environments.

(B) In the intestinal lumen, $V$. cholerae is exposed to bile acids, which repress expression of one PDE, VC1295, and activity of three DGCs; CdgH, CdgM, and VC1372. This regulation results in an overall increase in c-di-GMP level. Bicarbonate quenches the effect of bile acids on c-di-GMP, suggesting that c-di-GMP levels decrease near intestinal epithelial cells, where bicarbonate levels are higher. Polyamines are present in the human gastrointestinal tract. Norspermidine is bound by the periplasmic sensor protein NspS, which then enhances the PDE activity of MbaA. Spermidine triggers the opposite response, suggesting that it may compete with norspermidine for the NspS binding site. In classical $V$. cholerae strains, contact with host cells enhances expression of vieA, which leads to production of the PDE and critical virulence factor VieA. 


\section{Acknowledgements}

2 We thank Jennifer Teschler for her comments on the manuscript. Work in the laboratory

3 of FHY is supported by the US National Institute of Health (NIH) grants Al114261 and

4 Al102584. JC is supported by the National Science Foundation (NSF) Graduate

5 Research Fellowship. Work in the laboratory of HS is supported by the US National 6 Institute of Health (NIH) grant R01-Al097307. 


\section{References}

1. Galperin MY, Nikolskaya AN, Koonin E V.: Novel domains of the prokaryotic two-component signal transduction systems. FEMS Microbiol. Lett. 2001, 203:11-21.

2. Srivastava D, Hsieh M-L, Khataokar A, Neiditch MB, Waters CM: Cyclic di-GMP inhibits Vibrio cholerae motility by repressing induction of transcription and inducing extracellular polysaccharide production. Mol. Microbiol. 2013, 90:1262-1276.

** Identification of FlrA as a c-di-GMP binding receptor in $V$. cholerae.

3. Beyhan S, Tischler AD, Camilli A, Yildiz FH: Transcriptome and phenotypic responses of Vibrio cholerae to increased cyclic di-GMP level. J. Bacteriol. 2006, 188:3600-3613.

4. Liu X, Beyhan S, Lim B, Linington RG, Yildiz FH: Identification and characterization of a phosphodiesterase that inversely regulates motility and biofilm formation in Vibrio cholerae. J. Bacteriol. 2010, 192:4541-4552. ${ }^{*}$ Systematic identification of c-di-GMP metabolizing enzymes impacting motility in V. cholerae.

5. Beyhan S, Yildiz FH: Smooth to rugose phase variation in Vibrio cholerae can be mediated by a single nucleotide change that targets c-di-GMP signalling pathway. Mol. Microbiol. 2007, 63:995-1007.

*Systematic identification of c-di-GMP metabolizing enzymes impacting biofilm formation in $V$. cholerae.

6. Beyhan S, Odell LS, Yildiz FH: Identification and characterization of cyclic diguanylate signaling systems controlling rugosity in Vibrio cholerae. J. Bacteriol. 2008, 190:7392-405.

7. Satchell KJF, Jones CJ, Wong J, Queen J, Agarwal S, Yildiz FH: Phenotypic analysis reveals that the $\mathbf{2 0 1 0}$ Haiti cholera epidemic is linked to a hypervirulent strain. Infect. Immun. 2016, 84:2473-2481.

8. Pratt JT, McDonough E, Camilli A: PhoB regulates motility, biofilms, and cyclic di-GMP in Vibrio cholerae. J. Bacteriol. 2009, 191:6632-6642.

9. Beyhan $S$, Tischler $A D$, Camilli A, Yildiz FH: Differences in gene expression between the classical and EI Tor biotypes of Vibrio cholerae 01. Infect. Immun. 2006, 74:3633-3642.

10. Amikam D, Galperin MY: PilZ domain is part of the bacterial c-di-GMP binding protein. Bioinformatics 2006, 22:3-6.

11. Chou S, Galperin MY: Diversity of cyclic di-GMP-binding proteins and mechanisms. J. Bacteriol. 2016, 198:32-46.

12. Boehm A, Kaiser M, Li H, Spangler C, Kasper CA, Ackermann M, Kaever V, Sourjik V, Roth V, Jenal U: Second messenger-mediated adjustment of bacterial swimming velocity. Cell 2010, 141:107-116.

13. Paul K, Nieto V, Carlquist WC, Blair DF, Harshey RM: The c-di-GMP binding protein YcgR controls flagellar motor direction and speed to affect chemotaxis by a "backstop brake" mechanism. Mol. Cell 2010, 38:128-139.

14. Baker AE, Diepold A, Kuchma SL, Scott JE, Ha G, Orazi G, Armitage JP: PilZ domain protein FIgZ mediates cyclic di-GMP-dependent swarming motility 
control in Pseudomonas aeruginosa. J. Bacteriol. 2016, 198:1837-1846.

15. Gao X, Mukherjee S, Matthews PM, Hammad LA, Kearns DB, Dann CE: Functional characterization of core components of the Bacillus subtilis cyclic-Di-GMP signaling pathway. J. Bacteriol. 2013, 195:4782-4792.

16. Pratt JT, Tamayo R, Tischler AD, Camilli A: PilZ domain proteins bind cyclic diguanylate and regulate diverse processes in Vibrio cholerae. J. Biol. Chem. 2008, 148:825-832.

17. Roelofs KG, Jones CJ, Helman SR, Shang X, Orr MW, Goodson JR, Galperin MY, Yildiz FH, Lee VT: Systematic identification of cyclic-di-GMP binding proteins in Vibrio cholerae ra novel class of cyclic-di-GMP-binding ATPases associated with type II secretion systems. PLoS Pathog. 2015, 11:1-29. ** Systematic identification of c-di-GMP binding proteins in $V$. cholerae using novel assays.

18. Benach J, Swaminathan SS, Tamayo R, Handelman SK, Folta-Stogniew E, Ramos JE, Forouhar F, Neely H, Seetharaman J, Camilli A, et al.: The structural basis of cyclic diguanylate signal transduction by PilZ domains. EMBO J. 2007, 26:5153-5166.

19. Prouty MG, Correa NE, Klose KE: The novel sigma54- and sigma28-dependent flagellar gene transcription hierarchy of Vibrio cholerae. Mol. Microbiol. 2001, 39:1595-609.

20. Arora SK, Ritchings BW, Almira EC, Lory S, Ramphal R: A transcriptional activator, $\mathrm{FleQ}$, regulates mucin adhesion and flagellar gene expression in Pseudomonas aeruginosa in a cascade manner. J. Bacteriol. 1997, 179:55745581.

21. Matsuyama BY, Krasteva P V, Baraquet C, Harwood CS, Sondermann H, Navarro MVAS: Mechanistic insights into c-di-GMP-dependent control of the biofilm regulator FleQ from Pseudomonas aeruginosa. Proc. Natl. Acad. Sci. U. S. A. 2015, 113:E209-218.

** Reports the structure of FleQ in c-di-GMP bound state and Identified of c-diGMP binding motif of FleQ.

22. Krasteva P V, Fong JCN, Shikuma NJ, Beyhan S, Navarro MV a S, Yildiz FH, Sondermann $\mathrm{H}$ : Vibrio cholerae VpsT regulates matrix production and motility by directly sensing cyclic di-GMP. Science 2010, 327:866-888.

${ }^{* *}$ Reports the structure of VpsT in c-di-GMP bound state and identified of c-diGMP binding motif of VpsT.

23. Fong JCN, Yildiz FH: The rbmBCDEF gene cluster modulates development of rugose colony morphology and biofilm formation in Vibrio cholerae. $\mathrm{J}$. Bacteriol. 2007, 189:2319-2330.

24. Fong JCN, Karplus K, Schoolnik GK, Yildiz FH: Identification and characterization of $\mathrm{RbmA}$, a novel protein required for the development of rugose colony morphology and biofilm structure in Vibrio cholerae. J. Bacteriol. 2006, 188:1049-1059.

25. Teschler JK, Zamorano-Sánchez D, Utada AS, Warner CJA, Wong GCL, Linington RG, Yildiz FH: Living in the matrix: assembly and control of Vibrio cholerae biofilms. Nat. Rev. Microbiol. 2015, 13:255-268.

26. Shikuma NJ, Fong JCN, Yildiz FH: Cellular levels and binding of c-di-GMP 
control subcellular localization and activity of the Vibrio cholerae transcriptional regulator VpsT. PLoS Pathog. 2012, 8:e1002719.Shikuma NJ,

27. Zhao X, Koestler BJ, Waters CM, Hammer BK: Post-transcriptional activation of a diguanylate cyclase by quorum sensing small RNAs promotes biofilm formation in Vibrio cholerae. Mol. Microbiol. 2013, 89:989-1002.

28. Jones CJ, Utada A, Davis KR, Thongsomboon W, Zamorano-Sánchez D, Banakar V, Cegelski L, Wong GCL, Yildiz FH: C-di-GMP regulates motile to sessile transition by modulating MshA pili biogenesis and near-surface motility behavior in Vibrio cholerae. PLOS Pathog. 2015, 11:1-27.

${ }^{*}$ Identification of MshE as a c-di-GMP receptor controlling MSHA pili production.

29. Wang Y-C, Chin K-H, Tu Z-L, He J, Jones CJ, Sanchez DZ, Yildiz FH, Galperin MY, Chou S-H: Nucleotide binding by the widespread high-affinity cyclic diGMP receptor MshEN domain. Nat. Commun. 2016, 7:12481.

** Reports the structure of MshE in c-di-GMP bound state and Identified of c-diGMP binding motif of MshE.

30. Conner JG, Teschler JK, Jones CJ, Yildiz FH: Staying alive: Vibrio cholerae's cycle of environmental survival, transmission, and dissemination. Microbiol. Spectr. 2015, 2:1-32.

31. Zamorano-Sánchez D, Fong JCN, Kilic S, Erill I, Yildiz FH: Identification and characterization of VpsR and VpsT binding sites in Vibrio cholerae. J. Bacteriol. 2015, 197:1221-1235.

32. Srivastava D, Harris RC, Waters CM: Integration of cyclic di-GMP and quorum sensing in the control of vpsT and aphA in Vibrio cholerae. J. Bacteriol. 2011, 193:6331-6341.

33. Ayala JC, Wang H, Silva AJ, Benitez JA: Repression by H-NS of genes required for the biosynthesis of the Vibrio cholerae biofilm matrix is modulated by the second messenger cyclic diguanylic acid. Mol. Microbiol. 2015, 97:630-645.

34. Beyhan S, Bilecen K, Salama SR, Casper-Lindley C, Yildiz FH: Regulation of rugosity and biofilm formation in Vibrio cholerae: comparison of VpsT and VpsR regulons and epistasis analysis of vpsT, vpsR, and hapR. J. Bacteriol. 2007, 189:388-402.

35. Casper-lindley $\mathrm{C}$, Yildiz $\mathrm{FH}$ : VpsT is a transcriptional regulator required for expression of vps biosynthesis genes and the development of rugose colonial morphology in Vibrio cholerae 01 EI Tor. J. Bacteriol. 2004, 186:1574-1578.

36. Maclntyre DL, Miyata ST, Kitaoka M, Pukatzki S: The Vibrio cholerae type VI secretion system displays antimicrobial properties. Proc. Natl. Acad. Sci. U. S. A. 2010, 107:19520-19524.

37. Basler M, Mekalanos JJ: Type 6 secretion dynamics within and between bacterial cells. Science 2012, 337:1-4.

38. Pukatzki S, Ma AT, Revel AT, Sturtevant D, Mekalanos JJ: Type VI secretion system translocates a phage tail spike-like protein into target cells where it cross-links actin. Proc. Natl. Acad. Sci. U. S. A. 2007, 104:15508-15513.

39. Roth A, Breaker RR: The structural and functional diversity of metabolitebinding riboswitches. Annu. Rev. Biochem. 2009, 78:305-334. 
40. Dambach MD, Winkler WC: Expanding roles for metabolite-sensing regulatory RNAs. Curr. Opin. Microbiol. 2009, 12:161-169.

41. Sudarsan N, Lee ER, Weinberg Z, Moy RH, Kim JN, Link KH, Breaker RR: Riboswitches in eubacteria sense the second messenger cyclic di-GMP. Science 2008, 321:411-413.

** Identification of riboswitches as c-di-GMP receptors.

42. Lee ER, Baker JL, Weinberg Z, Sudarsan N, Breaker RR: An allosteric selfsplicing ribozyme triggered by a bacterial second messenger. Science 2010, 329:845-848.

43. Kirn TJ, Jude B a, Taylor RK: A colonization factor links Vibrio cholerae environmental survival and human infection. Nature 2005, 438:863-866.

44. Kariisa AT, Weeks K, Tamayo R: The RNA Domain Vc1 regulates downstream gene expression in response to cyclic diguanylate in Vibrio cholerae. PLOS One 2016, 11:1-17.

45. Metzger LC, Stutzmann S, Scrignari T, Van der Henst C, Matthey N, Blokesch M: Independent regulation of type VI secretion in by TfoX and TfoY. Cell Rep. 2016, 15:951-958.

* Revealed involvement of c-di-GMP in regulation of the type 6 secretion system.

46. Martinez-Wilson HF, Tamayo R, Tischler AD, Lazinski DW, Camilli A: The Vibrio cholerae hybrid sensor kinase VieS contributes to motility and biofilm regulation by altering the cyclic diguanylate level. J. Bacteriol. 2008, 190:6439-6447.

47. Mitchell SL, Ismail AM, Kenrick $S$ a, Camilli $A$ : The VieB auxiliary protein negatively regulates the VieSA signal transduction system in Vibrio cholerae. BMC Microbiol. 2015, 15:1-16.

48. Tischler $A D$, Lee $S H$, Camilli $A$ : The Vibrio cholerae vieSAB locus encodes a pathway contributing to cholera toxin production. J. Bacteriol. 2002, 184:4104-4113.

49. Dey AK, Bhagat A, Chowdhury R: Host cell contact induces expression of virulence factors and vieA, a cyclic di-GMP phosphodiesterase, in Vibrio cholerae. J. Bacteriol. 2013, 195:2004-2010.

50. Tamayo R, Schild S, Pratt JT, Camilli A: Role of cyclic di-GMP during El tor biotype Vibrio cholerae infection: characterization of the in vivo-induced cyclic di-GMP phosphodiesterase CdpA. Infect. Immun. 2008, 76:1617-1627.

51. Schild S, Tamayo R, Nelson EJ, Qadri F, Calderwood SB, Camilli A: Genes induced late in infection increase fitness of Vibrio cholerae after release into the environment. Cell Host Microbe 2007, 2:264-277.

52. Townsley L, Yildiz FH: Temperature affects c-di-GMP signaling and biofilm formation in Vibrio cholerae. Environ. Microbiol. 2015, 17:4290-4305.

53. Hung DT, Zhu J, Sturtevant D, Mekalanos JJ: Bile acids stimulate biofilm formation in Vibrio cholerae. Mol. Microbiol. 2006, 59:193-201.

54. Koestler BJ, Waters CM: Bile acids and bicarbonate inversely regulate intracellular cyclic di-GMP in Vibrio cholerae. Infect. Immun. 2014, 82:30023014.

* Impact of intestinal signals on cellular c-di-GMP levels were identified. 
55. Goforth JB, Walter NE, Karatan E: Effects of polyamines on Vibrio cholerae virulence properties. PLOS One 2013, 8:e60765.

56. Karatan E, Duncan TR, Watnick PI: NspS, a predicted polyamine sensor, mediates activation of Vibrio cholerae biofilm formation by norspermidine. J. Bacteriol. 2005, 187:7434-7443.

57. McGinnis MW, Parker ZM, Walter NE, Rutkovsky AC, Cartaya-Marin C, Karatan $\mathrm{E}$ : Spermidine regulates Vibrio cholerae biofilm formation via transport and signaling pathways. FEMS Microbiol. Lett. 2009, 299:166-174.

58. Cockerell SR, Rutkovsky AC, Zayner JP, Cooper RE, Porter LR, Pendergraft SS, Parker ZM, McGinnis MW, Karatan E: Vibrio cholerae NspS, a homologue of ABC-type periplasmic solute binding proteins, facilitates transduction of polyamine signals independent of their transport. Microbiology 2014, 160:832-843.

59. Schaller RA, Ali SK, Klose KE, Kurtz Jr. DM: A bacterial hemerythrin domain regulates the activity of a Vibrio cholerae diguanylate cyclase. Biochemistry 2013, 51:8563-8570.

${ }^{*}$ Mechanism of activation of a DGC is reported.

60. Orr MW, Donaldson GP, Severin GB, Wang J, Sintim HO, Waters CM, Lee VT:

Oligoribonuclease is the primary degradative enzyme for $p G p G$ in Pseudomonas aeruginosa that is required for cyclic-di-GMP turnover. Proc. Natl. Acad. Sci. U. S. A. 2015, 112:E5048-5057.

61. Cohen D, Mechold U, Nevenzal H, Yarmiyhu Y, Randall TE, Bay DC, Rich JD, Parsek MR, Kaever V, Harrison JJ, et al.: Oligoribonuclease is a central feature of cyclic diguanylate signaling in Pseudomonas aeruginosa. Proc. Natl. Acad. Sci. 2015, 112:11359-11364. 


\section{A}

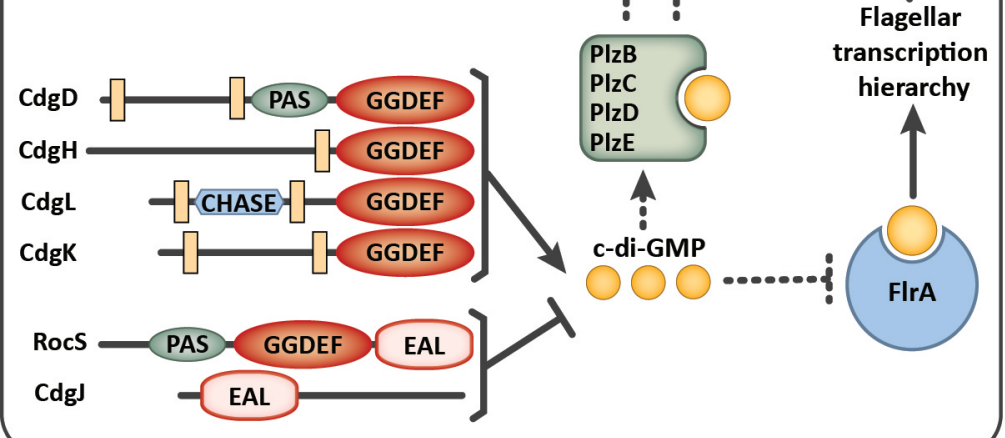

C

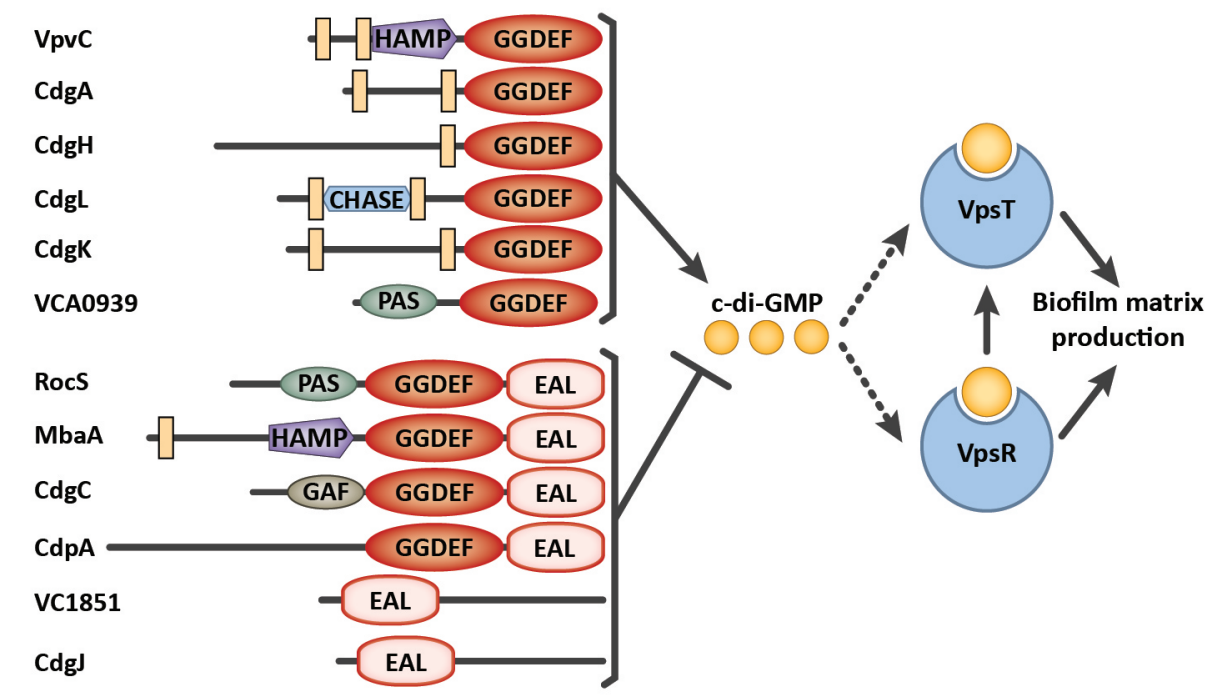

D

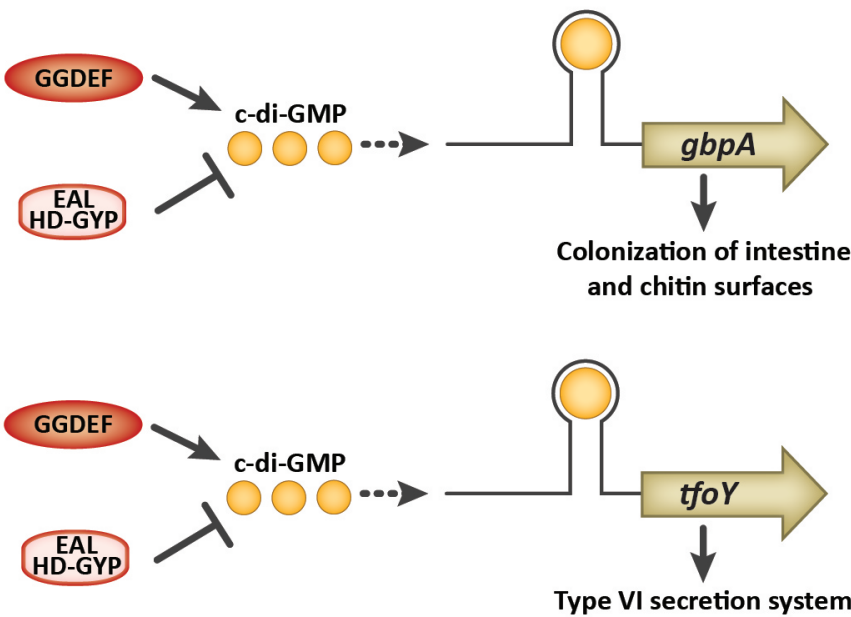

E

Codg

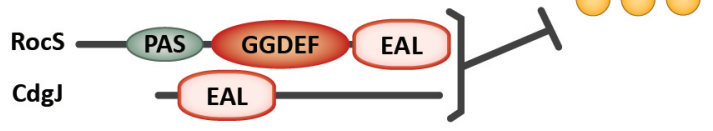

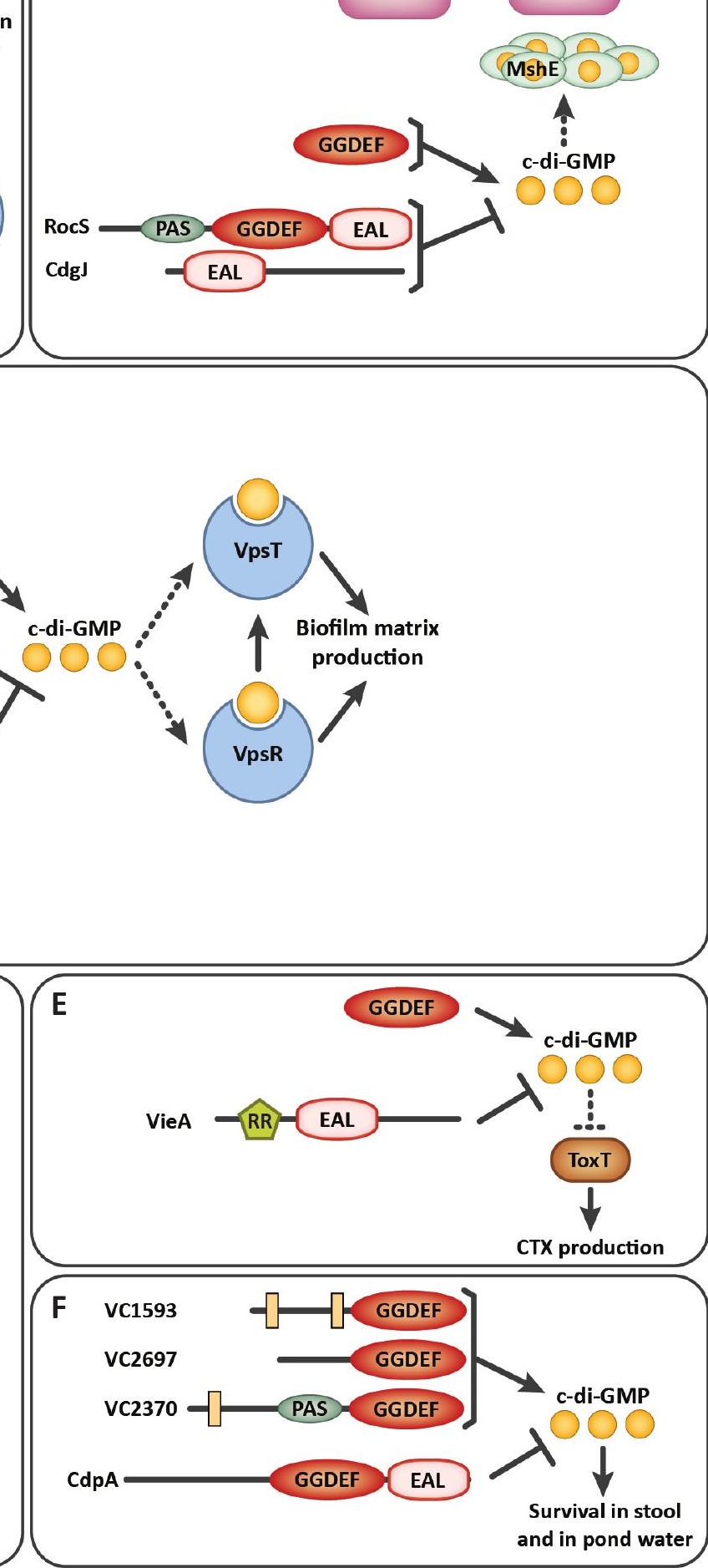




\section{A Vc Bhr-DGC}

Ferric Iron

High $\left[\mathrm{O}_{2}\right]$ Low $\left[\mathrm{O}_{2}\right]$ $\uparrow$
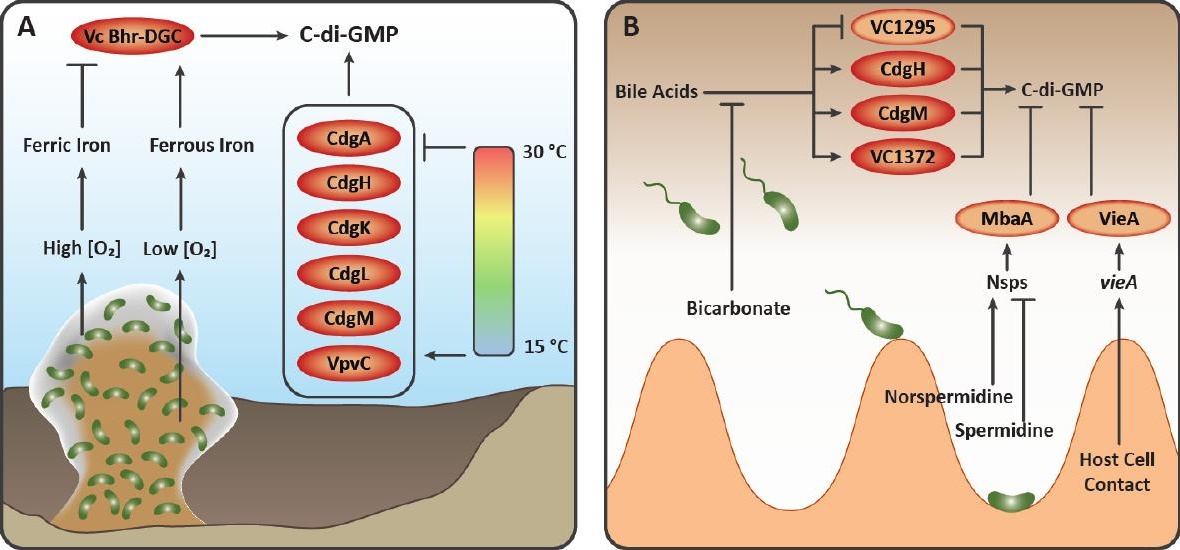

C-di-GMP

B rrous Iron
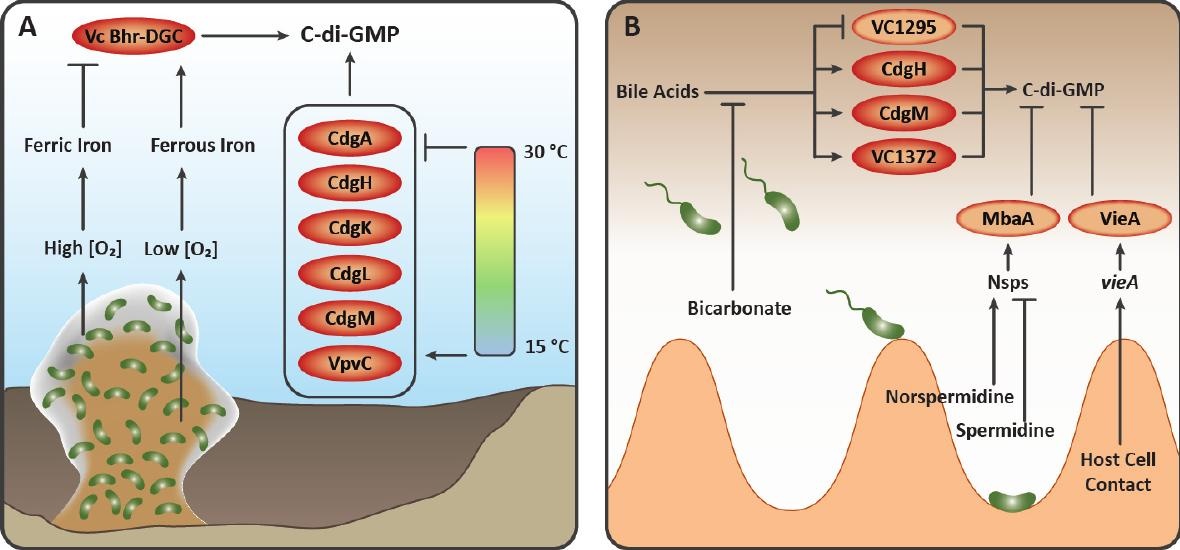

\section{Bicarbonate}

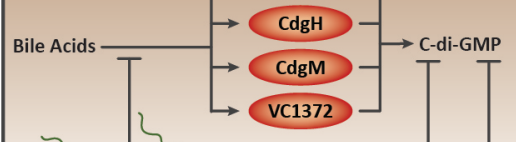

MbaA

Contact
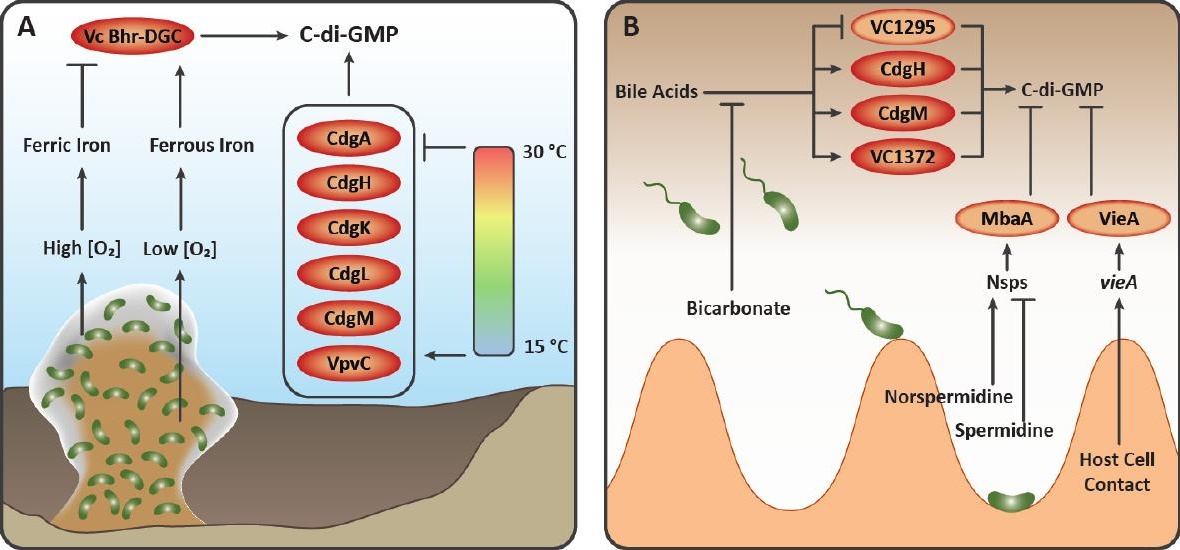


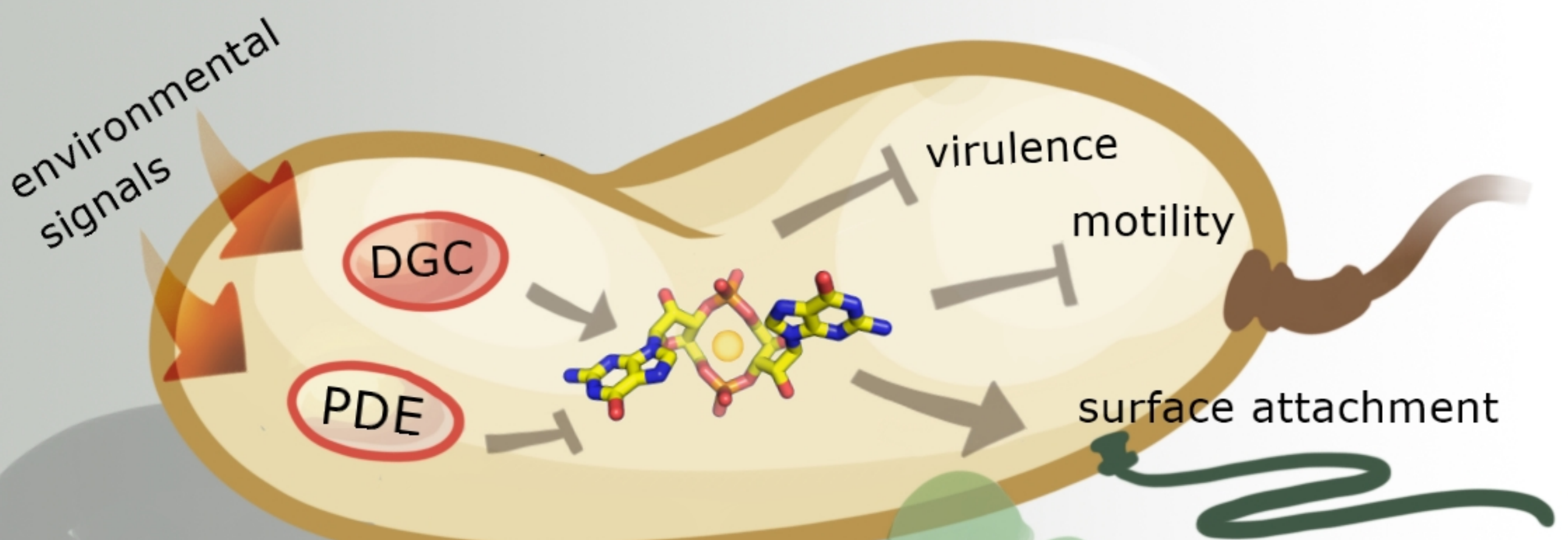

biofilm formation 